\title{
Next-Generation Counseling: Noninvasive Prenatal Screening Results Disclosure and
}

\section{Management}

Aishwarya Arjunan, MS, MPH, $\mathrm{CGC}^{1}$; email: aishwarya@counsyl.com

Rotem Ben-Shachar, $\mathrm{PhD}^{1}$; email: rbenschachar@counsyl.com

Jamie Kostialik, MS, CGC¹; email: jkostialik@counsyl.com

Katherine Johansen Taber, $\mathrm{PhD}^{1}$; email: kjohansentaber@counsyl.com

Gabriel Lazarin, MS, CGC ${ }^{1}$; email: gabriel@counsyl.com

Elizabeth Denne, MS, CGC'1 email: beth_denne@counsyl.com

Dale Muzzey, $\mathrm{PhD}^{1}$; email: dale@counsyl.com

Carrie Haverty, MS, CGC ${ }^{1}$ email: carrie@counsyl.com

${ }^{1}$ Counsyl, 180 Kimball Way, South San Francisco, CA 94080, USA

Conflict of Interest/Disclosure: All authors are employees of Counsyl.

Financial Support: This study was funded by Counsyl.

Corresponding Author:

Aishwarya Arjunan

180 Kimball Way

South San Francisco, CA 94080

T: 312-859-5003

F: 650-350-4092

E: research@counsyl.com 
Word Count: Abstract 344, Main text 2875

Condensation: A technology platform facilitates large-scale education, results delivery, and genetic counseling for noninvasive prenatal screening.

\section{Short title: Next-generation counseling for NIPS}

\section{A. Why was this study conducted?}

To determine the effectiveness of a technology platform in large-scale dissemination of noninvasive prenatal screening information and results.

\section{B. What are the key findings?}

A large-scale results delivery platform disseminating results, online education and genetic counseling was utilized by a diverse cohort of over 66,000 patients. Patients desired genetic counseling for both negative and positive results: approximately $7 \%$ of screen-negative patients and $19 \%$ of screen-positive patients elected genetic consultations, with $17 \%$ of screen-positive patients whose results were delivered by their provider requesting additional genetic counseling. The majority of patients requested on-demand genetic consultations.

\section{What does this study add to what is already known?}

This study is the first to show that a large-scale results delivery platform is effective in providing noninvasive prenatal screening results, education, and genetic counseling to patients. 


\begin{abstract}
Background: As noninvasive prenatal screening usage grows in the general obstetrics setting, proper patient education on the screen's benefits and limitations is needed.
\end{abstract}

Objective: Describe the use of a technology platform designed for large-scale dissemination of noninvasive prenatal screening information and results.

Study Design: The technology platform functioned as follows: Patients were emailed a link to an noninvasive prenatal screening general-education video upon laboratory receipt of a test requisition. Providers were then notified upon availability of patients' results. If noninvasive prenatal screening results were negative, the patient was sent an automated email with instructions to access results through a secure portal where she could watch tailored informational videos, request "on-demand" or scheduled genetic counseling, or decline any further services. If genetic counseling was elected, a summary of the session was sent to the ordering provider and patient upon completion. If noninvasive prenatal screening results were positive, either the ordering provider or a board-certified genetic counselor contacted the patient directly to communicate test results and provide counseling. The number and type of results issued through the platform, the number and type of genetic counseling consultations completed, and factors associated with requesting laboratory-delivered genetic counseling were tracked and analyzed for a 39-month period.

Results: Over the study period, 67,122 noninvasive prenatal screening results were issued through the platform, and 4,673 patients elected genetic counseling consultations; $95.2 \%$ $(n=4,450)$ of consultations were for patients receiving negative results. Over $70 \%(n=3,370)$ of consultations were on-demand rather than scheduled. Median consultation time was 14 minutes for positive results and six minutes for negative results. A positive screen, advanced maternal 
age, family history, previous history of a pregnancy with a chromosomal abnormality, and other high-risk pregnancy were associated with the greatest odds of electing laboratory-delivered genetic counseling.

Conclusions: By combining web education, automated notifications, and genetic counseling, we implemented a service that effectively facilitates results disclosure for ordering providers. These data demonstrate the capability to deliver noninvasive prenatal screening results, education, and counseling — congruent with management guidelines - to a large population, which is imperative to quality care as uptake increases.

Key Words: cell-free DNA analysis; genetic counseling; noninvasive prenatal screening; prenatal screening; results delivery; telehealth

\section{INTRODUCTION}

Noninvasive prenatal screening (NIPS) via cell-free DNA analysis represents a recent development in a decades-long history of fetal aneuploidy risk assessment. The utilization landscape has changed since its introduction in 2011, shifting from solely the high risk population to include many in the general prenatal population. ${ }^{1,2}$ As NIPS usage grows, proper patient education on its benefits and limitations is needed. The potential for misunderstanding the implications of test results creates the need to implement a scalable and robust protocol for results and education delivery. In a recent statement, the American College of Obstetricians and Gynecologists (ACOG) underscored the importance of communicating results to patients in a timely fashion and in the context of genetic counseling, adding that a policy of "no news is good news" (for negative test results) does not represent high quality care. ${ }^{3}$ 
Non-genetics providers play a critical role in educating patients about genetic testing, but they often lack confidence in their genetics knowledge, impacting their ability to have comprehensive discussions with their patients. ${ }^{4,5,6}$ This gap has been observed in many providers, including obstetricians and gynecologists. ${ }^{6,7}$ Therefore, additional mechanisms of providing genetic screening information and results are essential.

Software technologies offer potential solutions for efficient results delivery and genetic counseling. The integration of genomics and technology increasingly enables patient-provider communication. Patients are comfortable receiving health information online via patient portals rather than waiting one week or more for a provider to communicate test results, regardless of result type ${ }^{8}$ and genetic results delivery by a web-based platform has been found to be noninferior to return of results by an in-person genetic counselor. ${ }^{9}$ Other studies have shown that web-based education tools and telegenetic services are viewed as valuable by patients and providers, are effective in disseminating information to patients, and increase access to genetic clinicians while minimizing travel and reducing patient costs. ${ }^{10,11}$

This study describes the implementation of a service combining web education, automated results notifications, and genetic counseling that addresses two challenges: adequate education and results disclosure, and tracking of large-scale genetic testing in a methodical, robust, and timely fashion. We sought to explore whether this technology platform efficiently manages NIPS results disclosure with high patient utilization. 


\section{METHODS}

\section{Institutional Review Board Review}

This study was reviewed and designated as exempt by Western Institutional Review Board.

\section{Platform}

The software platform used in this study delivered education and results, and facilitated genetic counseling scheduling (Counsyl Complete ${ }^{\mathrm{TM}}$, Counsyl, South San Francisco, CA). The platform comprised two components: 1) a provider-facing Health Insurance Portability and Accountability Act (HIPAA)-compliant online portal that logged key events (e.g., completion of laboratory testing) and interactions between the patient and laboratory-employed genetic counselors, and 2) a patient-facing HIPAA-compliant portal that displayed test- and results-specific educational information and facilitated genetic counseling. Physician agreement was required to use the software platform. Results of all tests ordered were delivered through the platform, with exceptions described below.

\section{Automated Results Delivery System}

Genetic counselors employed by Counsyl utilized guidelines from ACOG, their own clinical expertise, and provider feedback to develop results notification, reminder, and tracking protocols. $^{12,13}$ American College of Medical Genetics and Genomics (ACMG) guidelines were utilized for the creation of post-test education and counseling elements in order to develop a protocol for the delivery of NIPS results. ${ }^{14}$ Figure 1 illustrates the automated results delivery system workflow. 


\section{Provider-facing Portal}

Ordering providers were notified via fax, email, or electronic medical record (EMR) upon results availability. The online portal contained an activity log of patient interactions, including all reminders sent throughout the results delivery process. Regardless of result type, if a patient elected a genetic counseling consultation, a report was sent to the ordering provider via fax, email, or EMR and was also available in the web portal.

\section{Patient-facing Portal}

Upon laboratory receipt of a test requisition, patients received an emailed link to a six-minute NIPS general education video created by genetic counselors at Counsyl in accordance with previously published recommendations. ${ }^{15}$ The website displayed detailed information regarding the conditions screened, as well as additional references and resources. Patients had the option of canceling the test at any point prior to release of results with no financial penalty.

\section{Negative Results}

Figure 1 describes the return of negative results to patients through the portal. Post-test education - presented in video and text format and accompanied by a downloadable clinical report—for screen-negative results summarized that no chromosomal abnormalities were detected, indicating a low residual risk for the tested conditions. All communication formats stated the possibility of false positive and false negative results. The clinical report included patient-specific residual risks for trisomies 13,18 , and 21 , and also stated the necessity of chorionic villus sampling (CVS) or amniocentesis if definitive diagnosis was desired. 


\section{Positive Results}

Screen-positive results were not automatically released to the patient (Fig. 1). Rather, the ordering provider's office was contacted by a genetic counselor and informed of the screenpositive result. The provider could opt to disclose the result to the patient directly via phone call or in-person appointment, through the portal, or by requesting that a genetic counselor contact the patient. All communication formats stated the possibility of false positive and false negative results and discussed individualized positive predictive value, when available. Similar to the reporting of screen-negative results, screen-positive results also stated the necessity of CVS or amniocentesis if definitive diagnosis was desired.

\section{Inconclusive Results}

A minority of results were of high complexity, such as no-calls due to sequencing error or suspected maternal aneuploidy. These were routed outside of the platform and were individually managed with the ordering provider. These results were not included in this study.

\section{Genetic Counseling}

The patient portal enabled patients to elect a consultation with a genetic counselor regardless of result type and at no additional cost. All genetic counselors were laboratory-employed, boardcertified, and licensed in the state of California, as well as licensed in the state in which they provided counseling, if required. Herein, when describing election of genetic counseling, we are referring specifically to the election of laboratory-delivered genetic counseling. 
For screen-positive results, patients could request a consultation even if the provider disclosed the results directly to the patient. A provider-facing activity log recorded whether or not a patient viewed their results and what type of counseling (on-demand or scheduled), if any, the patient elected. Patients requesting on-demand counseling were entered into a virtual queue and were contacted by telephone by a genetic counselor in the order the requests were received. Those requesting scheduled counseling could make an appointment for a future telephone consultation. The results delivery platform and education videos were in English, but certified medical interpreters for more than 200 languages were available if needed.

Following standard practice protocol and ACOG recommendations, genetic consultations included an overview of NIPS, a discussion of patient's results, and appropriateness of future diagnostic procedures. ${ }^{12,15}$ For patients who wished to pursue or further consider diagnostic testing, consultation with the ordering or other local provider was recommended. Consultation reports were made available to both the patient and provider upon completion. Patients were permitted to have unlimited sessions with no time limit, and counseling sessions were included in the cost of testing.

\section{Data Analysis}

Eligible patients' data were extracted from internal databases. Ethnicity was self-reported on the test requisition form. In order to be included in this analysis, the patient result necessarily was delivered through the automated results delivery system. Due to state regulations, samples from New York State were not included in data analyses. All statistical analyses were completed using Python version 2.7.13. Jeffrey's Bayesian interval and Goodman's method were used to compute 
binomial and multinomial proportion confidence intervals, respectively. For multivariate logistic regression analysis, we excluded from the analysis screen-positive patients that required a laboratory-administered genetic consultation $(n=32)$; a chi-squared test was used to calculate statistical significance for this analysis. A one-tailed proportion z-test was used to calculate whether the proportion of patients with positive test results that elected on-demand genetic counseling was significantly higher than that of patients with negative test results. A nonparametric Mann-Whitney test was used to determine statistical significance of differences in durations for genetic consultations for patients with negative versus positive test results.

\section{Noninvasive Prenatal Screen}

NIPS analyses were conducted at Counsyl (Prelude ${ }^{\mathrm{TM}}$ Prenatal Screen) or Illumina (Verifi, Illumina, San Diego, CA) using the whole-genome sequencing method described by Fan et al. ${ }^{17}$ Tests were ordered primarily by obstetricians, maternal-fetal medicine specialists, or genetics specialists. Patients from both high risk (e.g., advanced maternal age, other abnormal aneuploidy screen) and general prenatal populations were included. Chromosome analysis results could be reported as no aneuploidy detected ("negative"), aneuploidy detected ("positive"), or aneuploidy suspected (also "positive”).

\section{RESULTS}

\section{Cohort}

Over a 39-month period, 67,122 NIPS results were issued through the platform to 66,475 unique and eligible patients (Fig. 2). These results included 1,198 screen-positive tests, and 65,924 screen-negative tests. Of the 1,198 screen-positive results, $18.6 \%(n=223)$ of patients 
requested a genetic consultation. Median patient age was 34 years (interquartile range (IQR): 3037 years). Ethnicity was reported for 50,127 patients (75.4\%), and represented 14 different ethnicities (Supplementary Table 1).

The basic panel assessed aneuploidy risk for chromosomes 13,18 and 21 only $(n=2,946)$. In addition to screening for the basic panel, 57,654 screens assessed sex chromosome aneuploidy (SCA) risk (no microdeletions), 345 screens assessed microdeletions risk (no SCA), and 6,167 assessed both SCA and microdeletions risk. Median turnaround time for test results was 4 days (IQR: 3-5 days). Screen-positive result types are listed in Supplementary Table 2.

\section{Portal Use}

Results were successfully delivered to $99.7 \%(n=65,714)$ of patients who screened negative; remaining results were undeliverable due to incomplete or incorrect email addresses (Fig. 3). Of those receiving screen-negative results, $76.7 \%(n=50,547)$ viewed their test results in the portal and $6.75 \%(n=4,450)$ completed a genetic consultation (Fig. 3). Over $97 \%(n=1,166)$ of screenpositive results were delivered by the patient's provider. Providers requested that the laboratory deliver screen-positive results for 32 patients (2.67\%). More than $90 \%(n=29)$ of these individuals completed a genetic consultation; the remaining three $(9.38 \%)$ were unresponsive to requests for counseling. Of screen-positive patients whose provider delivered their results, $16.6 \%$ $(n=194)$ requested genetic counseling (Fig. 3). Eighty-seven percent of all screen-positive genetic consultations were for patients whose results were delivered by the provider.

\section{Factors affecting likelihood of laboratory-delivered genetic counseling}


Odds of choosing a genetic consultation were 11.9 times greater among those with screenpositive test results compared to those without a screen-positive test result $(\mathrm{P}<0.0001)$ (Fig. 4, Supplementary Table 3). Other significant factors associated with increased odds of electing genetic counseling included advanced maternal age (age at test of 35 years or older, both first and subsequent pregnancy), family history, history of a chromosomal abnormality in a previous pregnancy, and other high risk pregnancy $(\mathrm{P}<0.0001)$ (Fig. 4, Supplementary Table 3). Specific year of birth, whether a patient used in-vitro fertilization (IVF), and abnormal ultrasound were not significantly associated with increased odds of electing genetic counseling (Fig. 4). An ordering provider delivering test results was significantly associated with decreased odds of electing genetic counseling $(\mathrm{P}<0.0001)$.

\section{Consultations}

Of the total study population of 66,475 unique patients, 4,655 ( $7.0 \%$ overall; range of $4.2 \%$ $11.3 \%$ by ethnicity) elected genetic counseling. These 4,655 unique patients accounted for 4,673 total tests and 4,776 genetic consultations (Fig. 2), and had a median age of 35 years (IQR: 31-38 years). Median age among those who did not speak with a genetic counselor was 34 years (IQR: 30-37 years, $n=61,820$ ). Individuals of 14 ethnicities completed consultations (not shown). For 96 tests, multiple consultations were completed (Fig. 2 caption). The average wait time for patients seeking an on-demand genetic consultation was 11 minutes (IQR: 3-24 minutes).

An additional unassigned 242 genetic consultations were completed for 47 screen-positive results and 195 screen-negative results (Fig. 2). On-demand versus scheduled consultation status was not available for these 242 consultations as they occurred when a patient requested a genetic 
counseling appointment to discuss the results of a different test offered by the laboratory (e.g., carrier screening) and wished to discuss their NIPS result concurrently.

\section{Negative Results}

Of the 65,924 individuals with screen-negative test results, $6.75 \%(n=4,450)$ elected a genetic consultation. Consultations with individuals with negative screens accounted for $94.9 \%(n=$ $4,534)$ of all consultations; $70.4 \%(n=3,191)$ of these consultations were on-demand, and $25.3 \%$ $(n=1,148)$ were scheduled (Fig. 5a).

\section{Positive Results}

Genetic counseling was elected by $18.6 \%(n=223)$ of individuals with screen-positive results. Odds of a patient with a screen-positive test result choosing a genetic consultation were significantly higher than for a patient with a screen-negative result $(\mathrm{P}<0.0001)$. Of the consultations for screen-positive results, $74.0 \%(\mathrm{n}=179)$ were for on-demand genetic counseling, and $6.6 \%(n=16)$ were scheduled (Fig. 5a). A significantly higher proportion of patients with screen-positive test results sought on-demand counseling over scheduled counseling compared to patients with screen-negative test results $(\mathrm{P}<0.001)$.

\section{Consultation Durations}

Regardless of the type of consultation (scheduled versus on-demand), consultations for screenpositive test results had significantly longer durations than those for screen-negative test results $(\mathrm{P}<0.001)$ (Fig. 5b). The median consultation time for an individual with a positive screen was 
14 minutes (IQR: 10-20 minutes), whereas the median time for an individual with a negative screen was six minutes (IQR: 4-9 minutes) (Fig. 5b).

\section{COMMENT}

This study describes a results delivery platform that combined multiple media and communication formats to distribute a large number of NIPS results, while also facilitating education and genetic counseling. The platform is unique in several respects: it has served a large and diverse population of over 66,000 patients, has been in sustained clinical usage for three years, and demonstrates a previously uncommon level of involvement of the laboratory in results delivery, counseling, and patient education.

Patients have reported a high rate of satisfaction and comfort with telephone genetic counseling, ${ }^{18,19}$ and online education systems have been shown to have a positive impact on patient understanding and clinical outcomes. ${ }^{10,11,20}$ Web-based delivery platforms have also been tested and found to be noninferior to in-person counseling, representing an alternative method of educating patients about results. ${ }^{9}$ The desire for on-demand genetic counseling, regardless of result type, demonstrated in this study suggests that alternative counseling and education platforms will be necessary as genetic testing becomes more widespread in the clinical setting, and as practices attempt to follow guidelines recommending timely results delivery and counseling. ${ }^{3}$ Critically, clinicians have a responsibility to provide support, education, and counseling to patients when ordering testing. ${ }^{3}$ Laboratories offering testing have an opportunity to support this need by working with ordering providers to improve patient access to accurate, personalized, and timely information and counseling. As was the case in this study, many 
laboratories include genetic counseling in the cost of testing so that patients do not face affordability barriers to access counseling.

Our analyses show that although patients with positive results are most likely to elect genetic consultations, pre-existing risk factors, such as advanced maternal age, family history, and a previous pregnancy with a child affected with a chromosome abnormality, even among patients with negative results, were also associated with electing laboratory-provided genetic counseling. This suggests that all patients desire the option of genetic counseling; the counseling platform described in this study efficiently responds to such a desire. Additionally, a large number of patients with screen-positive results in our study elected a genetic consultation even after discussing results with a provider, demonstrating the desire for access to education and genetic counseling beyond that conducted by an ordering provider.

Compared to patients with screen-negative results, patients with screen-positive results demonstrated a significantly higher preference for on-demand genetic counseling versus a later scheduled appointment (Fig. 5a). However, this was true regardless of result type, suggesting that patients desire to receive education with results in a timely manner. Not surprisingly, median consultation duration was more than twice as long for patients with positive results versus those with negative results, likely because of the need to discuss diagnostic testing and other options in greater detail following a positive result. While alternative service delivery models such as the one described in this study offer patients and providers an opportunity to streamline workflow and increase access to post-test counseling, we note that pre-test counseling, not addressed in this study, is important for many patients. 
The study cohort included only patients whose providers chose to use the automated platform for NIPS results delivery and laboratory-based genetic counseling services. Therefore, we cannot definitively conclude that our results would be applicable to all prenatal patient populations. However, the cohort was large and diverse in terms of ethnicity and age, and was representative of our total tested population. This study did not collect data regarding patient motivators for using the platform, nor did it assess knowledge gain or retention among patients interacting with the portal. Pre-test counseling or other information provided by the ordering clinician may have impacted patient use of the portal or election of post-test counseling. In addition, observed effects of the service on the ordering providers' patient-management practices, such as reductions in time spent delivering results and providing post-test counseling, were not addressed in this study. These limitations lend themselves to important directions for future research.

By combining web education, automated notification protocols, and genetic counseling, we implemented a service that efficiently manages NIPS results disclosure. Providing large-scale results delivery, education, and counseling — congruent with clinical guidelines — is imperative to quality clinical care as genetic testing uptake grows among the general obstetric population. 
Acknowledgements: The authors thank Jaclyn Eng, and Harris Naemi for editorial assistance. Jaclyn and Harris are both employees of Counsyl. Good Publication Practice (GPP3) for manuscripts was followed when performing the research in this study and preparing the manuscript.

\section{REFERENCES}

1. Palomaki GE, Kloza EM, Lambert-Messerlian GM, et al. DNA sequencing of maternal plasma to detect Down syndrome: an international clinical validation study. Genet Med 2011;13:913-20.

2. Gregg AR, Skotko BG, Benkendorf JL, et al. Noninvasive prenatal screening for fetal aneuploidy, 2016 update: a position statement of the American College of Medical Genetics and Genomics. Genet Med 2016;18:1056-65.

3. American College of Obstetricians and Gynecologists. Counseling about genetic testing and communication of genetic test results. ACOG Practice Bulletin No. 693. Obstet Gynecol 2017;129:e96-101.

4. Klitzman R, Chung W, Marder K, et al. Attitudes and practices among internists concerning genetic testing. J Genet Couns 2013;22:90-100.

5. Houwink EJ, van Luijk SJ, Henneman L, van der Vleuten C, Jan Dinant G, Cornel MC. Genetic educational needs and the role of genetics in primary care: a focus group study with multiple perspectives. BMC Fam Pract 2011;12:5.

6. Mikat-Stevens NA, Larson IA, Tarini BA. Primary-care providers' perceived barriers to integration of genetics services: a systematic review of the literature. Genet Med 2015;17:169-76. 
7. Menzin AW, Anderson BL, Williams SB, Schulkin J. Education and experience with breast health maintenance and breast cancer care: a study of obstetricians and gynecologists. J Cancer Educ 2010;25:87-91.

8. Johnson AJ, Easterling D, Nelson R, Chen MY, Frankel RM. Access to radiologic reports via a patient portal: clinical simulations to investigate patient preferences. J Am Coll Radiol 2012;9:256-63.

9. Biesecker BB, Lewis KL, Umstead KL, et al. Web platform vs in-person genetic counselor for return of carrier results from exome sequencing: a randomized clinical trial. JAMA Intern Med 2018;178:338-46.

10. Lewis D. Computer-based approaches to patient education: A review of the literature. $\mathbf{J}$ Am Med Inform Assoc 1999;6:272-82.

11. Otten E, Birnie E, Ranchor AV, van Langen IM. Online genetic counseling from the providers' perspective: counselors' evaluations and a time and cost analysis. Eur J Hum Genet 2016;24:1255-61.

12. American College of Obstetricians and Gynecologists. Invasive prenatal testing for aneuploidy. ACOG Practice Bulletin No. 88. Obstet Gynecol 2007;110:e1459-67.

13. American College of Obstetricians and Gynecologists. Tracking and reminder systems. ACOG Committee Opinion No. 461. Obstet Gynecol 2010;116:e464-66.

14. Gregg AR, Gross SJ, Best RG, et al. ACMG statement on noninvasive prenatal screening for fetal aneuploidy. Genet Med 2013;15:395-98.

15. Sachs A, Blanchard L, Buchanan A, Norwitz E, Bianchi DW. Recommended pre-test counseling points for noninvasive prenatal testing using cell-free DNA: a 2015 perspective. Prenat Diagn. 2015;35(10):968-71. 
16. American College of Obstetricians and Gynecologists. Prenatal diagnostic testing for genetic disorders. ACOG Practice Bulletin No. 162. Obstet Gynecol 2016;127:e108-22.

17. Fan HC, Blumenfeld YJ, Chitkara U, Hudgins L, Quake SR. Noninvasive diagnosis of fetal aneuploidy by shotgun sequencing DNA from maternal blood. Proc Natl Acad Sci USA 2008;105:16266-71.

18. Schwartz MD, Valdimarsdottir HB, Peshkin BN, et al. Randomized noninferiority trial of telephone versus in-person genetic counseling for hereditary breast and ovarian cancer. $\mathrm{J}$ Clin Oncol 2014;32:618-26.

19. Sutphen R, Davila B, Shappell H, et al. Real world experience with cancer genetic counseling via telephone. Fam Cancer 2010;9:681-89.

20. Kuppermann M, Pena S, Bishop JT, et al. Effect of Enhanced Information, Values Clarification, and Removal of Financial Barriers on Use of Prenatal Genetic Testing. JAMA 2014;312(12):1210-1217.

\section{FIGURE LEGENDS}

Figure 1. Automated results delivery workflow: Providers are notified upon results availability. If results are screen-negative, patients are contacted and reminded to access results and educational resources, as well as request genetic counseling, through the portal. If results are screen-positive, providers are contacted by the laboratory about preferred method of results delivery: 1.) the provider may request that the laboratory inform the patient and provide counseling, or 2.) the provider may inform the patient directly and release results to the patient through the portal, and advise the patient to schedule a genetic consultation with the laboratory. Regardless of result type, if genetic counseling is elected, a consultation report is sent to the ordering provider via fax, email, or EMR. 


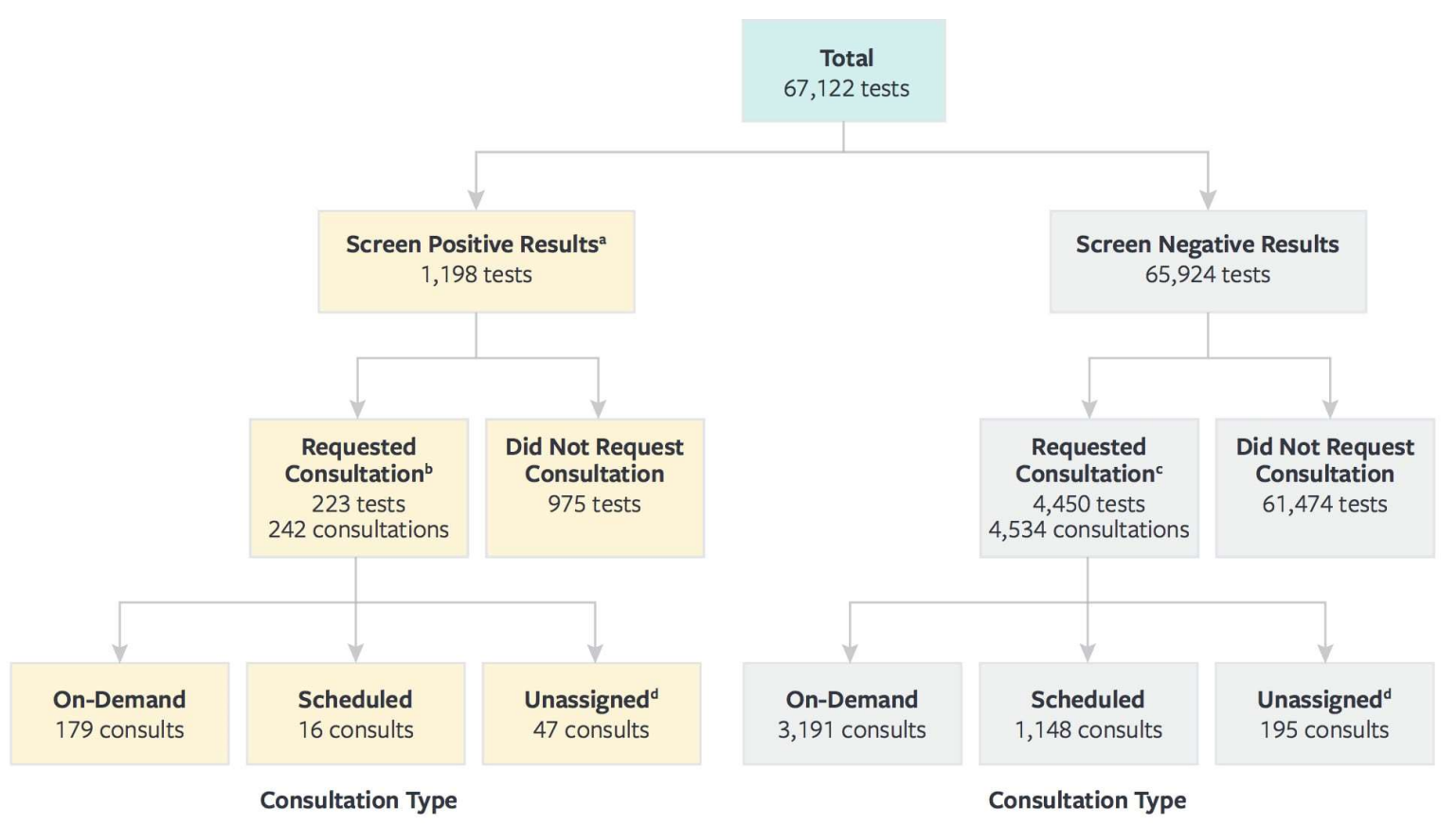

Figure 2. Results delivered and consultation types scheduled through the automated

delivery platform. ${ }^{\text {a }}$ Screen-positive results include both aneuploidy suspected and aneuploidy detected. ${ }^{b}$ For screen-positive results, 204 patients had one consultation and 19 had two consultations. ${ }^{c}$ For screen-negative results, 4373 patients had one consultation, 70 had two consultations, and seven had three consultations. ${ }^{\mathrm{d} O n-d e m a n d ~ v e r s u s ~ s c h e d u l e d ~ c o n s u l t a t i o n ~}$ status was unassigned for 47 screen-positive and 195 screen-negative consultations as they occurred when a patient requested a genetic counseling appointment to discuss the results of different test offered by Counsyl (e.g., carrier screening) and also wished to discuss their NIPS result. 


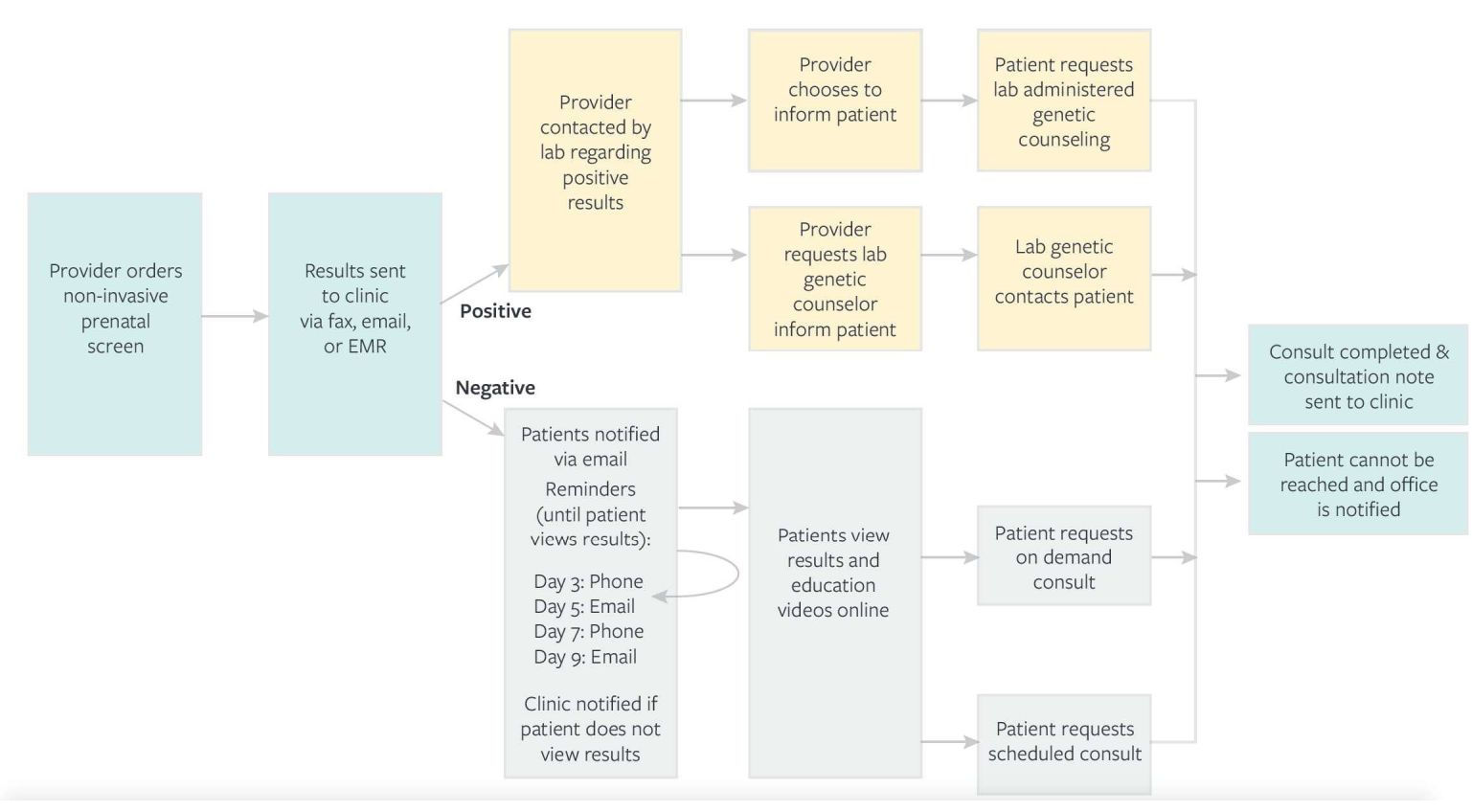

Figure 3. Patient utilization of automated results workflow. Results delivery for screennegative and screen-positive results, with positive screens stratified by provider and laboratory delivery. In each horizontal bar, shading denotes the percentage of patients with the specified result type that completed the action denoted in the automated results workflow described in Figure 1. The pink bar denotes screen-negative results, stratified by results released (light pink), patient viewing of results (medium pink), and laboratory-delivered genetic counseling (dark pink). The solid green bar denotes screen-positive results delivered by the laboratory, stratified by the percentage of patients that did (dark green) and did not (light green) elect genetic counseling. The hatched green bar denotes screen-positive results delivered by the provider, stratified by the percentage of patients that did (dark green) and did not (light green) elect genetic counseling. 95\% confidence intervals are shown. 


\section{RESULTS DELIVERED BY LAB:}

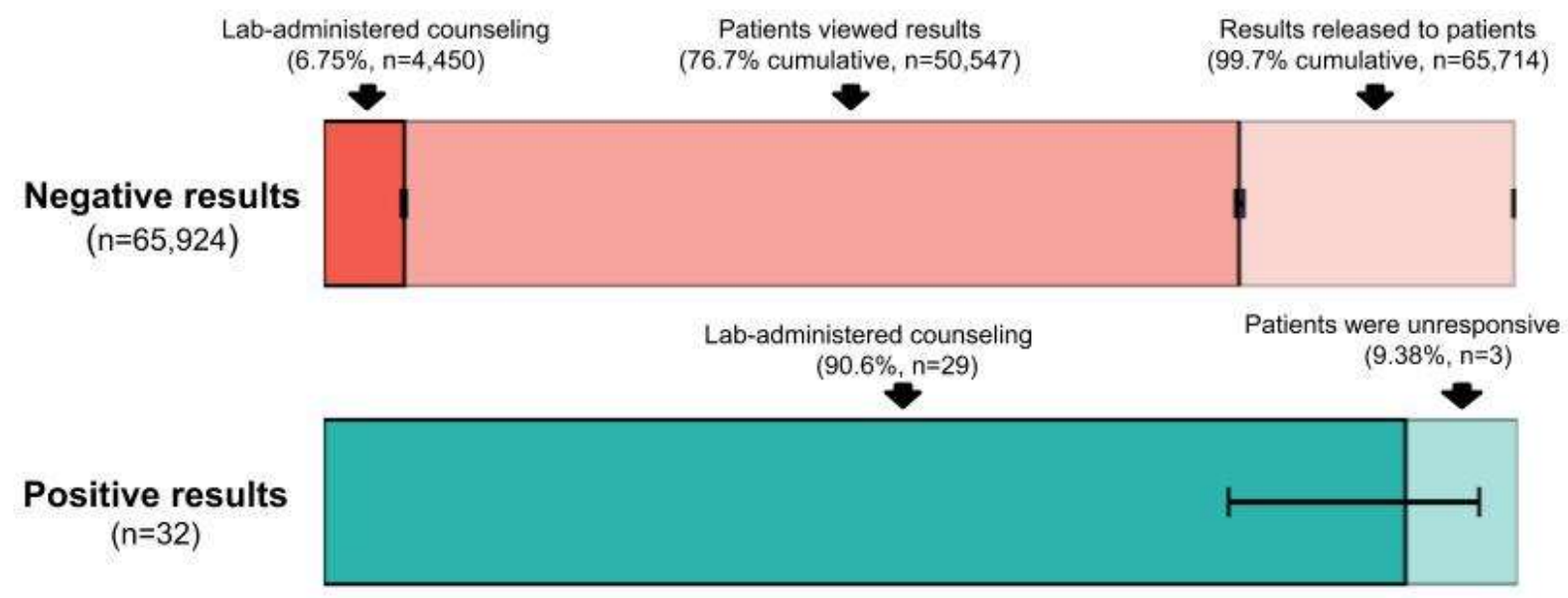

\section{POSITIVE RESULTS DELIVERED BY PROVIDER:}

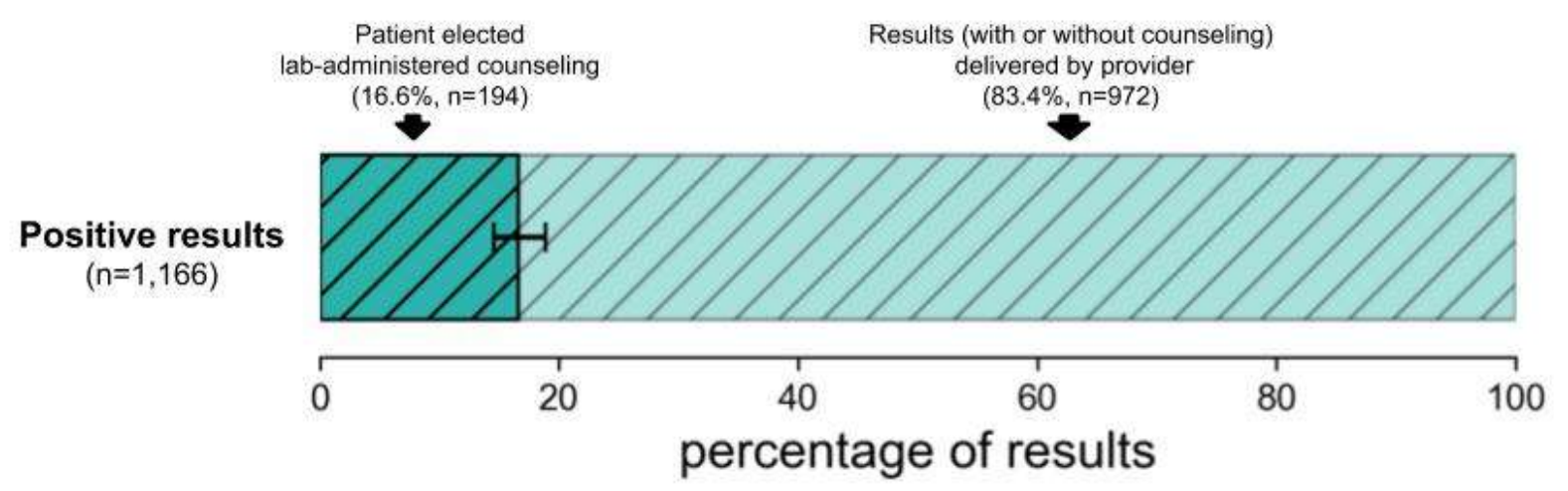

Figure 4. Factors most associated with electing laboratory-delivered genetic counseling. 
Odds ratios of factors associated with higher propensity of seeking laboratory-delivered genetic counseling. An odds ratio greater than one indicates that a patient with the factor of interest is at increased odds to elect genetic counseling. An odds ratio less than one indicates that a patient with the factor of interest is at decreased odds to elect genetic counseling. Circles show point estimates of odds ratios. $95 \%$ confidence intervals are shown with horizontal lines. Statistical significance is shown with stars. ****: $\mathrm{P}<0.0001$; ***: $\mathrm{P}<0.001$; ** $\mathrm{P}<0.01 ; * *$ : $\mathrm{P}<0.05$; n.s: not significant at the $\mathrm{p}=0.05$ significance level. AMA: advanced maternal age, U/S: ultrasound, CNS: central nervous system, IVF: in vitro fertilization. 


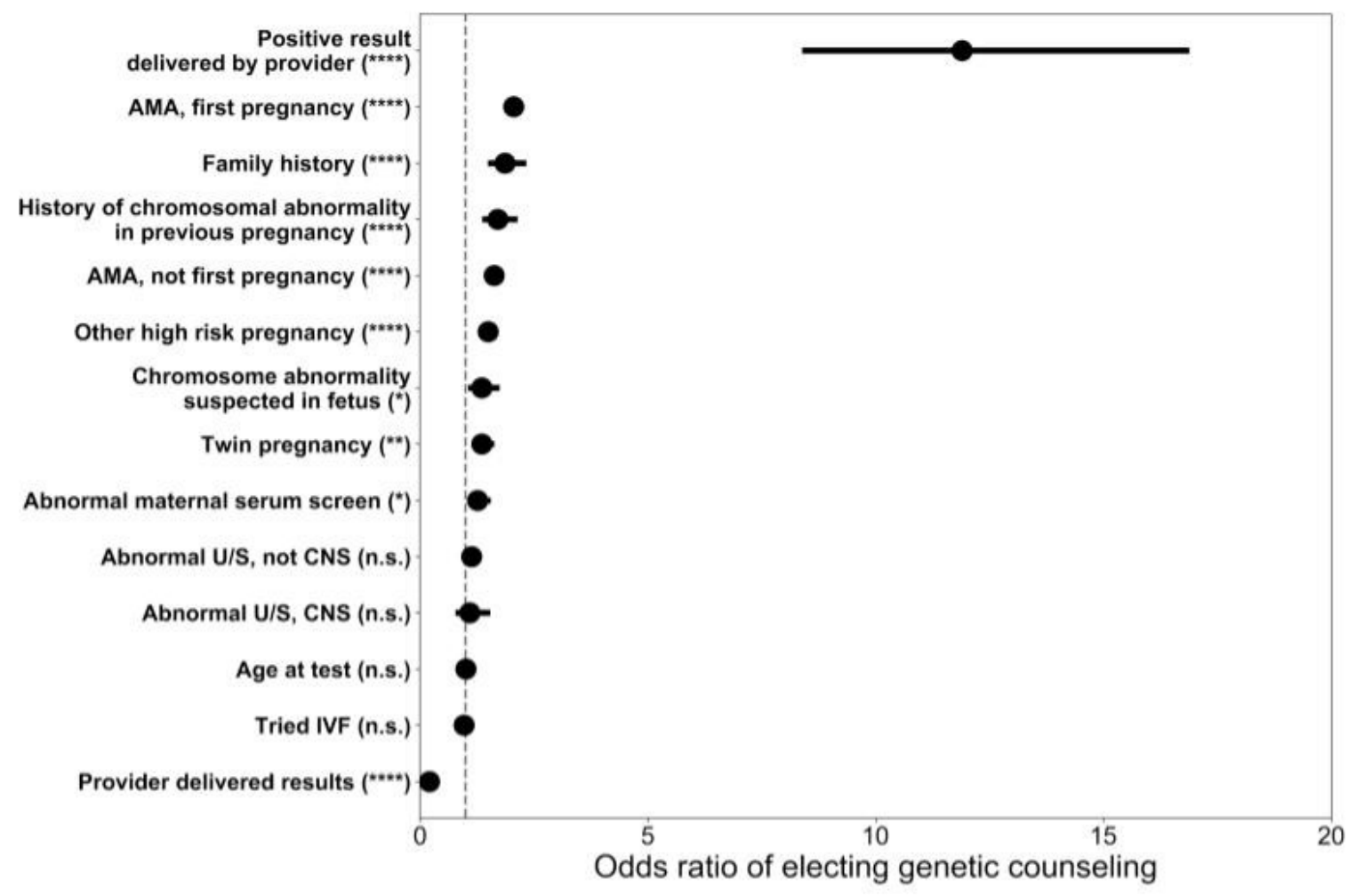


Figure 5. Utilization of genetic counseling. (a) Fraction of on-demand and scheduled genetic counseling sessions, stratified by result type (screen-negative or screen-positive), with $95 \%$ confidence intervals. Unassigned consultations are not reflected. (b) Consultation durations stratified by reservation type and result type. Consultation duration times are significantly lower for screen-negative results compared to screen-positive results at the $\mathrm{P}<0.001$ significance level (indicated by “****”) for both on-demand and scheduled consults. Bolded lines show median values and the boxes show interquartile (IQR) range. Vertical lines show 1.5 times the IQR. For (a) and (b), 242 unassigned consultations ( $n=47$ screen positive results and $n=195$ screen negative results) are not included. 
(a)

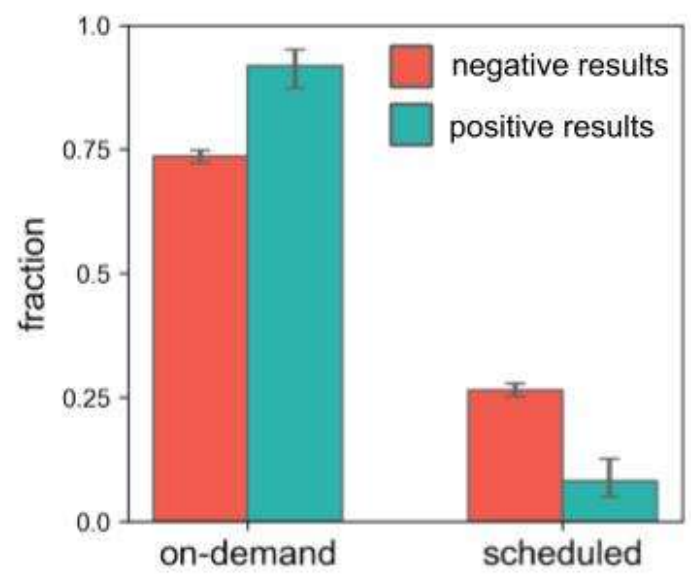

(b)

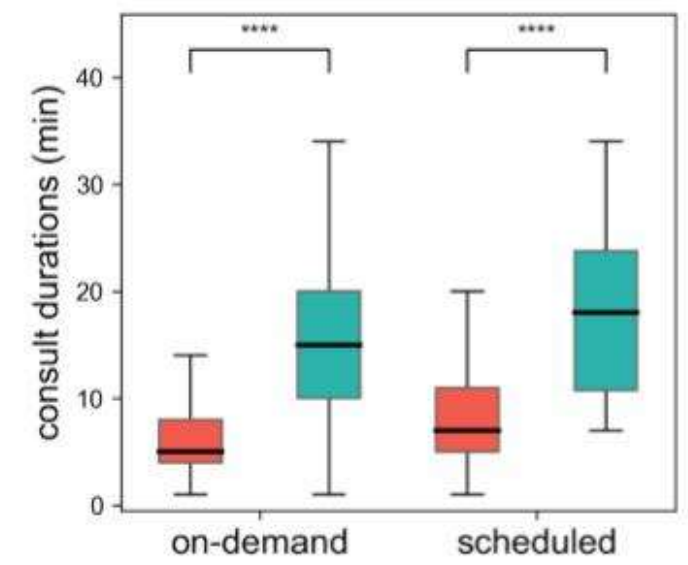


